\title{
Hyperfine effects on potassium tune-out wavelengths and polarizabilities
}

\author{
Jun Jiang and J. Mitroy \\ School of Engineering, Charles Darwin University, Darwin NT 0909, Australia
}

(Dated: October 7, 2018)

\begin{abstract}
The influence of hyperfine interactions on the tune-out wavelengths of the ${ }^{39,40,41} \mathrm{~K}$ isotopes of the potassium atom was investigated. The hyperfine interaction of the $4 s_{1 / 2}$ ground state results in a shift and splitting of the primary tune-out wavelength near $769 \mathrm{~nm}$. The $4 s_{1 / 2}$ state hyperfine splittings of the primary tune-out wavelength were almost equal to the hyperfine splittings of the ground states. The splittings in the wavelengths were $0.0008,0.0027$ and $0.0005 \mathrm{~nm}$ for ${ }^{39} \mathrm{~K},{ }^{40} \mathrm{~K}$ and ${ }^{41} \mathrm{~K}$ respectively. The hyperfine splitting of the $n p_{J}$ levels leads to the creation of additional tune-out wavelengths. The additional tune-out wavelengths could be difficult to detect due to very small differences from the transition wavelengths to the $4 p_{J, F}$ states. The hyperfine Stark shift for the ground states of all three isotopes were also computed and the value for ${ }^{30} \mathrm{~K}$ was found to be compatible with the previous experiments and the most recent calculation using relativistic many body perturbation theory.

PACS numbers: 31.15.ac, 31.15.ap, 34.20.Cf
\end{abstract}

\section{INTRODUCTION}

The primary tune-out wavelength for the $4 s_{1 / 2}$ ground state of neutral potassium located between the $4 p_{1 / 2}$ and $4 p_{3 / 2}$ excitations has recently measured to be $768.9712(15) \mathrm{nm}$ [1]. There have also been calculations of a number of tune-out wavelengths for potassium [2, 3]. The present work extends those calculations to take into consideration the impact of hyperfine interactions on the tune-out wavelengths for the ${ }^{39,40,41} \mathrm{~K}$ isotopes. Plans to measure tune-out wavelengths to increased precision mean that hyperfine splitting could easily become significant at the level of anticipated precision [1, 4]. The present article determines the impact of the hyperfine structure upon the tune-out wavelengths of potassium. We use the matrix elements previously used to determine the tune-out wavelengths of potassium [3] and minimal details of this previous calculation are presented.

The concept of the tune-out wavelength was initially introduced as a means to release one atom species from a two species optical lattice [5]. Other applications of tuneout wavelengths within two species optical lattices [2] have recently been discussed. One of possible application would be the measurements of tune-out wavelengths to make precise estimates of oscillator strength ratios [2]. A relative precision of about $0.2 \%$ has been achieved in the $5 s \rightarrow 6 p_{J}$ transition matrix elements of rubidium 6 and a relative precision of $0.19 \%$ has been achieved for the $\left(4 s \rightarrow 4 p_{1 / 2}\right):\left(4 s \rightarrow 4 p_{3 / 2}\right)$ line strength ratio for potassium [1]. There are relatively few atomic oscillator strengths measured at this level of precision [7].

The present investigation entails the determination of the polarizability of the $\mathrm{K}(4 s)$ ground state for different hyperfine levels. The calculations are similar to those used in the determination of the hyperfine Stark shift and there have been a number of experiments and calculations on hyperfine Stark shifts for the alkali atoms [8-14]. The most important of these investigations are those related to the determination of the blackbody ra- diation shift of the ${ }^{137}$ Cs clock transition 14].

The approach used to determine the influence of the hyperfine shift upon the dynamic polarizability is somewhat unorthodox. Most calculations of the hyperfine Stark shift are performed using third order perturbation theory $13-16$. The hyperfine interaction operator alters the polarizability in two distinctly different ways. First of all, the hyperfine energy shifts directly enter the denominator in the oscillator strength sum rule used to calculate the dipole polarizability. Second, the hyperfine interactions leads to the mixing of the ground $4 s_{1 / 2}$ state with excited bound $n s_{1 / 2}$ and continuum $\varepsilon_{1 / 2}$ states. This results in a change of the $4 s_{1 / 2} \rightarrow 4 p_{3 / 2}$ line strengths. These two effects have roughly same influence on the polarizability.

The approach used to calculate the hyperfine polarizabilities utilizes existing information about the hyperfine interaction constants to determine the hyperfine energy shifts of the low lying states of potassium. We do not use a perturbation theory calculation to determine the impact of the hyperfine interaction on the line strength. Instead, the dipole matrix element from the resonant transition is treated as a parametric function of the binding energy, and matrix elements adjusted for the hyperfine energy shifts are used in the calculation of the polarizability. The method was validated by a comparison with the previous calculated and experimental hyperfine Stark shifts for ${ }^{39} \mathrm{~K}[11,15-18]$. Shifts in the primary tune-out wavelengths for the ground states of ${ }^{39,40,41} \mathrm{~K}$ were found to be very closely related to the hyperfine energy shifts of the ground states.

\section{FORMULATION AND CALCULATIONS}

\section{A. Hyperfine splitting}

The first part of our calculation required the determination of the hyperfine energy shifts of the ground and 
low-lying excited states. Rather than calculate this information directly, the information is sourced from experiment or other calculations. The potassium dipole polarizability is dominated by the resonant transition, so hyperfine splitting is only taken into consideration for the ground state and some of the low-lying excited states.

According to first-order perturbation theory, the energy for a hyperfine state $|L J I F\rangle$ is given [19, 20] by

$$
E=E_{N L J}+W_{F}
$$

where $E_{N L J}$ is the energy for the unperturbed fine structure state and $W_{F}$ is the hyperfine interaction energy. The hyperfine interaction energy can be written as

$$
W_{F}=\frac{1}{2} A R+B \frac{\frac{3}{2} R(R+1)-2 I(I+1) J(J+1)}{2 I(2 I-1) 2 J(2 J-1)}
$$

where,

$$
R=F(F+1)-I(I+1)-J(J+1),
$$

$F$ is total angular momentum, $I$ is the nuclear spin, $J$ is the total electronic angular momentum of the associated fine structure state, and $A$ and $B$ are hyperfine structure constants. It is usual to give the $A$ and $B$ coefficients in $\mathrm{MHz}$ where $1.0 \mathrm{MHz}=1.519829903 \times 10^{-10}$ a.u. The energies of the different hyperfine states of ${ }^{39} \mathrm{~K},{ }^{40} \mathrm{~K}$ and ${ }^{41} \mathrm{~K}$ are listed in Table I. The ${ }^{39} \mathrm{~K}$ and ${ }^{41} \mathrm{~K}$ isotopes are stable, while ${ }^{40} \mathrm{~K}$ has a very long lifetime. The ${ }^{39} \mathrm{~K}$ isotope is the most common at $93.3 \%$ abundance. These tabulations are only include the $4 s_{1 / 2}, 4 p_{J}$ and $5 p_{J}$ states. The hyperfine energy shifts are largest for ${ }^{40} \mathrm{~K}$. The energy shifts for the $4 s_{1 / 2}$ ground states are about an order of magnitude larger than those of the $4 p_{1 / 2}$ excited states. Similarly, the hyperfine splitting for the $n p_{1 / 2}$ states are significantly larger than the splitting of the $n p_{3 / 2}$ states.

\section{B. Reduced Matrix Elements}

The dipole matrix elements between the different hyperfine levels are computed from the original $L J$ tabulation [3] using the Wigner-Eckart theorem. The original calculations of the potassium tune-out wavelengths were explicitly relativistic. These matrix elements, after making a small energy dependent correction described later, were then used to compute the polarizabilities and subsequently the tune-out wavelengths.

The transition matrix elements between the two hyperfine levels $\left|n_{1} L_{1} J_{1} I F_{1}\right\rangle$ and $\left|n_{2} L_{2} J_{2} I F_{2}\right\rangle$ can be written as

$$
\begin{aligned}
& \left\langle L_{2} J_{2} I F_{2}\left\|r^{k} C^{k}(r)\right\| L_{1} J_{1} I F_{1}\right\rangle=(-1)^{I+J_{2}+F_{1}+k} \\
\times & \hat{F}_{1} \hat{F}_{2}\left\{\begin{array}{ccc}
I & J_{1} & F_{1} \\
k & F_{2} & J_{2}
\end{array}\right\}\left\langle L_{2} J_{2}\left\|r^{k} C^{k}(r)\right\| L_{1} J_{1}\right\rangle
\end{aligned}
$$

where $k=1$ for a dipole transition and $\hat{F}=\sqrt{2 F+1}$.
The absorption oscillator strength $f_{g i}^{(k)}$ for a dipole transition from hyperfine level $g \rightarrow i$ is defined in the $F$-representation as

$$
f_{g i}^{(k)}=\frac{2\left|\left\langle L_{i} J_{i} I F_{i}\left\|r^{k} C^{k}(r)\right\| L_{g} J_{g} I F_{g}\right\rangle\right|^{2} \varepsilon_{g i}}{(2 k+1)\left(2 F_{g}+1\right)}
$$

where $\varepsilon_{g i}$ is the excitation energy of the transition.

The matrix elements, $A_{i j}$, are treated as parametric functions of their binding energies. The functional form adopted is

$$
\begin{aligned}
A_{i j}\left(E_{i}, E_{j}\right) & \approx A_{i j}\left(E_{0, i}, E_{0, j}\right)+\frac{\partial A_{i j}}{\partial E_{i}}\left(E_{i}-E_{0, i}\right) \\
& +\frac{\partial A_{i j}}{\partial E_{j}}\left(E_{i}-E_{0, j}\right)
\end{aligned}
$$

where $E_{0, i}$ and $E_{0, j}$ are the binding energies without any hyperfine splitting. The original $(L, J)$ matrix element set was computed using a single-electron approach with a semi-empirical polarization potential [3]. The partial derivatives were evaluated by redoing the calculations with a slightly different polarization potential and noting the change in the reduced matrix elements. The original $4 s \rightarrow 4 p_{J}$ and $4 s \rightarrow 5 p_{J}$ matrix elements of the $(L, J)$ calculation and the matrix element derivatives are listed in Table

\section{Handling isotopic effects}

Calculations of polarizabilities and tune-out wavelengths have also been done for the ${ }^{40,41} \mathrm{~K}$ isotopes. The

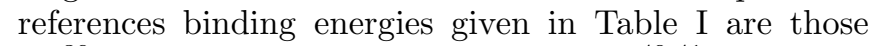
of ${ }^{39} \mathrm{~K}$. The energies attributed to the ${ }^{40,41} \mathrm{~K}$ isotopes in Table \ were computed as follows. The energies for the $\mathrm{K}(5 p)$ states were used as a reference point and held fixed. The overall binding energies of the ${ }^{40,41} \mathrm{~K}(4 s)$ and ${ }^{40,41} \mathrm{~K}\left(4 p_{J}\right)$ states were then adjusted to be more tightly bound by including the isotope shifts listed in Table III. The binding energies given in Table $\llbracket$ for the $\mathrm{K}(4 s)$ and $\mathrm{K}\left(4 p_{J}\right)$ states incorporate these shifts. It is necessary to include these shifts since tune-out wavelengths depend sensitively on the energy spacing of the $\mathrm{K}\left(n p_{J}\right)$ levels from the ground state.

While the separation between the $\mathrm{K}\left(4 s, 4 p_{J}, 5 p_{J^{\prime}}\right)$ levels is correct for the different isotopes, the absolute binding energies of these levels cannot be guaranteed to be correct for ${ }^{40,41} \mathrm{~K}$. This has implications for the relative polarizabilities of the different isotopes, but has little impact upon the determination of the polarizability differences of the hyperfine levels within the same isotope. 
TABLE I: The binding energies of the hyperfine states of ${ }^{39} \mathrm{~K},{ }^{40} \mathrm{~K}$ and ${ }^{41} \mathrm{~K}$ relative to the $\mathrm{K}^{+}$core. The nuclear spin, $I$ is indicated. The notation $a[b]$ means $a \times 10^{b}$. The energies of the different isotopes are normalized so that the energies of the $5 p_{J}$ levels are the same. Hyperfine interaction constants are sourced from experiment with two exceptions which are denoted by labelling with $(\mathrm{T})$. The absolute binding energies are known to eight significant digits so the digits beyond that should only be interpreted as having significance when comparing the energy differences of the hyperfine levels of the same isotope.

\begin{tabular}{|c|c|c|c|c|c|c|}
\hline & $E_{n L J}$ (a.u.) & $A(\mathrm{MHz})$ & $B(\mathrm{MHz})$ & $F$ & $W_{F}$ (a.u.) & $E_{n L J I F}$ (a.u.) \\
\hline & & & ${ }^{39} \mathrm{~K}, I=1 / 2$ & & & \\
\hline \multirow[t]{2}{*}{$4 s_{1 / 2}$} & -0.15951645 & 230.859860 [19] & - & 1 & $-4.3858[-8]$ & -0.159516493858 \\
\hline & & & & 2 & $2.6315[-8]$ & -0.159516423685 \\
\hline \multirow{2}{*}{$4 p_{1 / 2}$} & -0.10035159 & 27.775 [21] & - & 1 & $-5.2767[-9]$ & -0.100351595277 \\
\hline & & & & 2 & $3.1660[-9]$ & -0.100351586834 \\
\hline \multirow[t]{4}{*}{$4 p_{3 / 2}$} & -0.100088643 & $6.093[21]$ & $2.786[21]$ & 0 & $-2.9433[-9]$ & -0.100088645943 \\
\hline & & & & 1 & $-2.4407[-9]$ & -0.100088645441 \\
\hline & & & & 2 & $-1.0121[-9]$ & -0.100088644012 \\
\hline & & & & 3 & $2.1894[-9]$ & -0.100088640811 \\
\hline \multirow[t]{2}{*}{$5 p_{1 / 2}$} & -0.0469686695 & $-9.02[19]$ & - & 1 & $-1.7136[-9]$ & -0.0469686712136 \\
\hline & & & & 2 & $1.0281[-9]$ & -0.0469686684718 \\
\hline \multirow[t]{5}{*}{$5 p_{3 / 2}$} & -0.0468832095 & $-1.969[19]$ & $-0.87[19]$ & 0 & $-9.5692[-10]$ & -0.0468832104569 \\
\hline & & & & 1 & $-7.8989[-10]$ & -0.0468832102899 \\
\hline & & & & 2 & $-3.2361[-10]$ & -0.0468832098236 \\
\hline & & & & 3 & $7.0638[-10]$ & -0.0468832087936 \\
\hline & & & ${ }^{40} \mathrm{~K}, I=4$ & & & \\
\hline \multirow[t]{2}{*}{$4 s_{1 / 2}$} & -0.15951648572 & -285.7308 [19] & - & $7 / 2$ & $1.0857[-7]$ & -0.159516377150 \\
\hline & & & & $9 / 2$ & $-8.6852[-8]$ & -0.159516572568 \\
\hline \multirow[t]{2}{*}{$4 p_{1 / 2}$} & -0.100351606621 & $-34.52300[21]$ & - & $7 / 2$ & $1.3117[-8]$ & -0.100351593504 \\
\hline & & & & $9 / 2$ & $-1.0494[-8]$ & -0.100351617115 \\
\hline \multirow[t]{4}{*}{$4 p_{3 / 2}$} & -0.10008865956 & $-7.585[21]$ & $-3.445[21]$ & $5 / 2$ & $8.3888[-9]$ & -0.100088651173 \\
\hline & & & & $7 / 2$ & $4.7140[-9]$ & -0.100088654848 \\
\hline & & & & $9 / 2$ & $-3.4733[-10]$ & -0.100088659909 \\
\hline & & & & $11 / 2$ & $-7.0476[-9]$ & -0.100088666610 \\
\hline \multirow{2}{*}{$5 p_{1 / 2}$} & -0.0469686695 & $-10.98(\mathrm{~T})[22]$ & - & $7 / 2$ & $4.1719[-9]$ & -0.0469686653281 \\
\hline & & & & $9 / 2$ & $-3.3375[-9]$ & -0.0469686728375 \\
\hline \multirow[t]{5}{*}{$5 p_{3 / 2}$} & -0.0468832095 & $-2.45[19]$ & $-1.16[19]$ & $5 / 2$ & $2.7061[-9]$ & -0.0468832067939 \\
\hline & & & & $7 / 2$ & $1.5240[-9]$ & -0.0468832079759 \\
\hline & & & & $9 / 2$ & $-1.0904[-10]$ & -0.0468832096090 \\
\hline & & & & $11 / 2$ & $-2.2782[-9]$ & -0.0468832117782 \\
\hline & & & ${ }^{41} \mathrm{~K}, I=3 / 2$ & & & \\
\hline \multirow[t]{2}{*}{$4 s_{1 / 2}$} & -0.1595165190 & 127.0069352 [19] & - & 1 & $-2.4129[-8]$ & -0.159516543159 \\
\hline & & & & 2 & $1.4477[-8]$ & -0.159516504553 \\
\hline \multirow[t]{2}{*}{$4 p_{1 / 2}$} & -0.1003516232 & $15.245[21]$ & - & 1 & $-2.8962[-9]$ & -0.100351626136 \\
\hline & & & & 2 & $1.7377[-9]$ & -0.100351621502 \\
\hline \multirow[t]{4}{*}{$4 p_{3 / 2}$} & -0.1000886761 & $3.362[21]$ & $3.351[21]$ & 0 & $-1.2795[-9]$ & -0.100088677420 \\
\hline & & & & 1 & $-1.2778[-9]$ & -0.100088677418 \\
\hline & & & & 2 & $-7.6520[-10]$ & -0.100088676905 \\
\hline & & & & 3 & $1.2770[-9]$ & -0.100088674863 \\
\hline \multirow[t]{2}{*}{$5 p_{1 / 2}$} & -0.0469686695 & $-4.84(\mathrm{~T})[22]$ & - & 1 & $-9.1950[-10]$ & -0.0469686704195 \\
\hline & & & & 2 & $5.5169[-10]$ & -0.0469686689483 \\
\hline \multirow[t]{4}{*}{$5 p_{3 / 2}$} & -0.0468832095 & $-1.08[19,23]$ & $1.06[19,23]$ & 0 & $-4.1415[-10]$ & -0.0468832099142 \\
\hline & & & & 1 & $-4.1111[-10]$ & -0.0468832099111 \\
\hline & & & & 2 & $-2.4393[-10]$ & -0.0468832097439 \\
\hline & & & & 3 & $4.0959[-10]$ & -0.0468832090904 \\
\hline
\end{tabular}

\section{Dipole polarizability}

The dynamic dipole polarizabilities can be computed with the usual oscillator strength sum-rules. This is
The sum over $i$ includes all allowable fine-structure and hyperfine-structure allowed transitions. The $\omega \rightarrow 0$ limit of Eq. (7) is the static dipole polarizability. The dipole polarizability also has a tensor component for states with

$$
\alpha_{1}(\omega)=\sum_{i} \frac{f_{g i}^{(1)}}{\varepsilon_{g i}^{2}-\omega^{2}}
$$


TABLE II: The partial derivatives describing the variation of the matrix elements with respect to the initial and final state binding energies.

\begin{tabular}{lccc}
\hline \hline Transition & $A\left(E_{0 i}, E_{0 j}\right)$ & $\frac{\partial A}{\partial E_{4 s}}$ & $\frac{\partial A}{\partial E_{j}}$ \\
\hline $4 s_{1 / 2}-4 p_{1 / 2}$ & 4.102991192 & 28.334 & -0.548 \\
$4 s_{1 / 2}-4 p_{3 / 2}$ & 5.801566158 & 40.258 & -1.267 \\
$4 s_{1 / 2}-5 p_{1 / 2}$ & 0.2633449165 & -17.058 & 138.399 \\
$4 s_{1 / 2}-5 p_{3 / 2}$ & 0.3886341459 & -23.872 & 193.610 \\
\hline \hline
\end{tabular}

TABLE III: Isotope shifts, $\delta \nu$, of the $4 s-4 p_{1 / 2}, 4 s-4 p_{3 / 2}$ and $4 s-5 p$ transitions of potassium. The shifts are for the mean energies once the effects of hyperfine splitting have been removed. The positive shifts mean that the transition energy would be be larger for ${ }^{40,41} \mathrm{~K}$ than for ${ }^{39} \mathrm{~K}$. The notation $a[b]$ means $a \times 10^{b}$.

\begin{tabular}{|c|c|c|}
\hline & $(\mathrm{MHz})$ & (a.u.) \\
\hline & $\overline{\mathrm{IS}}\left(4 s-4 p_{1 / 2}\right)[21]$ & \\
\hline$\delta \nu\left({ }^{40} \mathrm{~K}-{ }^{39} \mathrm{~K}\right)$ & $125.64(10)$ & $1.9095(16)[-8]$ \\
\hline \multirow{2}{*}{$\delta \nu\left({ }^{41} \mathrm{~K}-{ }^{39} \mathrm{~K}\right)$} & $235.49(9)$ & $3.5790(14)[-8]$ \\
\hline & $\mathrm{IS}\left(4 s-4 p_{3 / 2}\right)[21]$ & \\
\hline$\delta \nu\left({ }^{40} \mathrm{~K}-{ }^{39} \mathrm{~K}\right)$ & $126.03(15)$ & $2.0674(23)[-8]$ \\
\hline \multirow{2}{*}{$\delta \nu\left({ }^{41} \mathrm{~K}-{ }^{39} \mathrm{~K}\right)$} & $236.18(17)$ & $3.5895(25)[-8]$ \\
\hline & $\operatorname{IS}(4 s-5 p)[24]$ & \\
\hline$\delta \nu\left({ }^{40} \mathrm{~K}-{ }^{39} \mathrm{~K}\right)$ & $235.0(20)$ & $3.572(36)[-8]$ \\
\hline$\delta \nu\left({ }^{41} \mathrm{~K}-{ }^{39} \mathrm{~K}\right)$ & $454.2(8)$ & $6.903(14)[-8]$ \\
\hline
\end{tabular}

TABLE IV: The scalar, $\alpha_{1}$, and tensor, $\alpha_{1}^{T}$, dipole polarizabilities of the hyperfine states of ${ }^{39,40,41} \mathrm{~K}$. The notation $a[b]$ means $a \times 10^{b}$. The absolute precision of the polarizabilities is about $1 \%$ but polarizability differences of different hyperfine states of the same isotope should be accurate to better than $10^{-5}$ a.u.

\begin{tabular}{lccc}
\hline \hline State & $F$ & $\alpha_{1}$ (a.u.) & $\alpha_{1}^{T}$ (a.u.) \\
\hline${ }^{39} \mathrm{~K} 4 s_{1 / 2}$ & 1 & 290.0493839 & $0.102[-5]$ \\
${ }^{39} \mathrm{~K} 4 s_{1 / 2}$ & 2 & 290.0499846 & $-0.481[-5]$ \\
${ }^{40} \mathrm{~K} 4 s_{1 / 2}$ & $7 / 2$ & 290.0505970 & $-0.103[-4]$ \\
${ }^{40} \mathrm{~K} 4 s_{1 / 2}$ & $9 / 2$ & 290.0489242 & $-0.166[-4]$ \\
${ }^{41} \mathrm{~K} 4 s_{1 / 2}$ & 1 & 290.0493810 & $0.668[-6]$ \\
${ }^{41} \mathrm{~K} 4 s_{1 / 2}$ & 2 & 290.0497114 & $-0.242[-5]$ \\
\hline \hline
\end{tabular}

TABLE V: The hyperfine Stark shifts of the ground states of ${ }^{39,40,41} \mathrm{~K}$. The notation $a[b]$ means $a \times 10^{b}$.

\begin{tabular}{lc}
\hline \hline Method & $\Delta \alpha_{1}$ (a.u.) \\
\hline & ${ }^{39} \mathrm{~K}: \alpha_{1}(F=2)-\alpha_{1}[(F=1)$ \\
Present & $6.007[-4]$ \\
MBPT-SD [1] & $5.996[-4]$ \\
Perturbation theory [15] & $4.9[-4]$ \\
Perturbation theory [16] & $5.49[-4]$ \\
Experiment [17] & $6.11(61)[-4]$ \\
Experiment [18] & $5.7(2)[-4]$ \\
\hline & \\
Present & ${ }^{40} \mathrm{~K}: \alpha_{1}(F=9 / 2)-\alpha_{1}(F=7 / 2)$ \\
Present & $1.673[-3]$ \\
\hline \hline
\end{tabular}

$F>1 / 2$. This can be written

$$
\begin{aligned}
\alpha_{1}^{\mathrm{T}}(\omega) & =6\left(\frac{5 F_{g}\left(2 F_{g}-1\right)\left(2 F_{g}+1\right)}{6\left(F_{g}+1\right)\left(2 F_{g}+3\right)}\right)^{1 / 2} \\
& \times \sum_{i}(-1)^{F_{g}+F_{i}}\left\{\begin{array}{ccc}
F_{g} & 1 & F_{i} \\
1 & F_{g} & 2
\end{array}\right\} \frac{f_{g i}^{(1)}}{\varepsilon_{g i}^{2}-\omega^{2}}
\end{aligned}
$$

Static scalar and tensor dipole polarizabilities for the potassium isotope ground states are listed in Table IV. The polarizability for a state with non-zero angular momentum $F_{g}$ depends on the magnetic projection $M_{g}$, these can be calculated using the formula [8],

$$
\alpha_{1, M_{g}}(\omega)=\alpha_{1}(\omega)+\alpha_{1}^{T}(\omega) \frac{3 M_{g}^{2}-F_{g}\left(F_{g}+1\right)}{F_{g}\left(2 F_{g}-1\right)},
$$

and numerical values are given in the supplementary data.

Table $\nabla$ gives the hyperfine Stark shift, i.e. the difference between the polarizabilities for two states with the same $(L, J)$ but different $F$ quantum numbers. There have been some previous investigations of the hyperfine Stark shift for ${ }^{39} \mathrm{~K}$ [11, 15 18]. The polarizability differences in Table $\mathrm{V}$ are given in a.u. The hyperfine Stark shift is often reported experimentally as a Stark shift coefficient $k$, with units of $\left(\mathrm{Hz} /(\mathrm{V} / \mathrm{m})^{2}\right.$. This is converted into a.u. by multiplying by $0.4018778 \times 10^{8}$ [8]. The present hyperfine Stark shift, is in very good agreement with the value from a singles plus doubles all-order relativistic many body perturbation theory calculation 11 and about $5 \%$ different than the experimental values 17, 18]. Differences with earlier calculations are hard to assess since useful data such as the resonant oscillator strength were not given [15, 16].

The changes to the dipole matrix elements were an important part of the polarizability calculation. The polarizability difference between the $F=1$ and $F=2$ states of ${ }^{39} \mathrm{~K}$ was $6.007 \times 10^{-4}$ a.u. However, omitting the matrix element correction resulted in a hyperfine Stark shift of about half this size, namely of $3.306 \times 10^{-4}$ a.u. The changes in the polarizabilities for $F=1$ and $F=2$ states of ${ }^{39} \mathrm{~K}$ due to change in the matrix elements were $-1.688 \times 10^{-4}$ a.u. and $1.013 \times 10^{-4}$ a.u., respectively.

The polarizabilities in Table IV need to be interpreted properly. The overall uncertainty in the polarizability is larger than the precision to which the values have been reported would imply. The uncertainty is about $1 \%$ [3, 8, 25]. However, the polarizability differences between different hyperfine states are essentially perturbative calculations despite the fact the current methodology involves the calculation of two polarizabilities followed by a subtraction. So the hyperfine Stark shifts can be given to an accuracy of at least $10^{-4}$ a.u. even though the overall precision in the calculated polarizabilities are closer to 1 a.u.

Table $\mathrm{V}$ also gives the hyperfine Stark shifts for the ground states of ${ }^{40} \mathrm{~K}$ and ${ }^{41} \mathrm{~K}$. There does not appear to be any experimental or theoretical information on the 
hyperfine Stark shifts for either of these isotopes. The hyperfine Stark shift for ${ }^{40} \mathrm{~K}$ is close to three times larger than the hyperfine Stark shift for ${ }^{39} \mathrm{~K}$. This is a consequence of the larger hyperfine interaction constants of ${ }^{40} \mathrm{~K}$. The ${ }^{41} \mathrm{~K}$ isotope has the smallest hyperfine interaction constants and the smallest Stark shift.

The calculations of the polarizabilities for ${ }^{40,41} \mathrm{~K}$ isotopes take into account the isotope shifts (IS) of the $4 s$, $4 p_{J}$ and $5 p_{J}$ states. The energy intervals between these levels incorporate the isotope shifts into the polarizability calculation. However, matrix elements were not adjusted to take into account the overall isotope shift. This means that not much significance can be attributed to the differences in the polarizabilities of the potassium ground states for the different isotopes. These could be estimated if the absolute shift in the ionization energies of these three isotopes were known. However, the isotope shifts of the ground state ionization energies are not known so the isotope shift in polarizabilities of the ground states cannot be estimated. But to reiterate, the present methodology does allow for the difference in polarizabilities due to hyperfine structure to be estimated correctly.

All of the potassium isotopes have a non-zero nuclear spin, so all of the ground states will have a tensor polarizability which is listed in Table IV. The ${ }^{40} \mathrm{~K}$ isotope, with the largest nuclear spin, has the largest tensor polarizabilities. But even for this system, the tensor polarizability does not exceed $2 \times 10^{-5}$ a.u. in magnitude. The differences between the polarizabilities for the different magnetic sub-levels of the hyperfine states did not exceed $2 \times 10^{-5}$ a.u. Polarizabilities for the different hyperfine magnetic sub-levels are listed in the supplementary data.

\section{E. Tune-out wavelengths}

$$
\text { 1. }{ }^{39} K
$$

The tune out wavelengths for the two hyperfine levels of the $4 s_{1 / 2}$ ground state of ${ }^{39} \mathrm{~K}$ are listed in Table VI. Hyperfine splitting leads to two new features in the tune-out spectrum. First of all, the splitting of the $4 s_{1 / 2}$ ground state into two hyperfine levels has resulted in two duplicate sets of tune-out wave lengths, one for the $4 s_{1 / 2, F=1}$ state and one for the $4 s_{1 / 2, F=2}$ state. The tune-out wavelengths are given to eight digits after the decimal point to ensure all the differences between all hyperfine generated tune-out wavelengths are given to at least two digits.

The hyperfine splitting of the $4 p_{J, F}$ levels has also resulted in the creation of additional tune-out wavelengths that arise when the polarizability contributions from two adjacent hyperfine levels act to cancel each other. Figure 1 is a schematic energy level diagram showing the location of all the tune-out wavelengths for the ${ }^{39} \mathrm{~K} 4 s_{1 / 2,1}$ state. The hyperfine splitting of the $4 p_{1 / 2, F}$ states has resulted in one additional tune-out wavelength, located be-

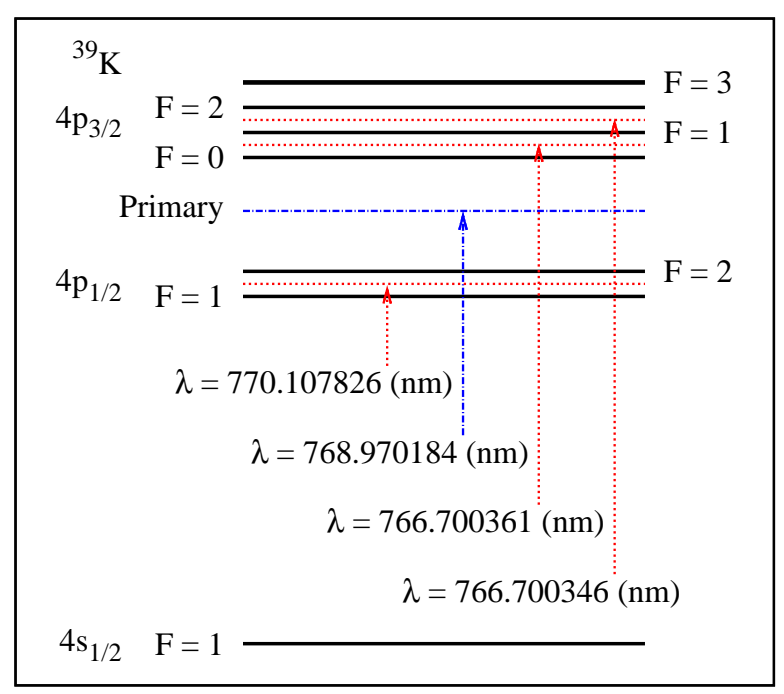

FIG. 1: (color online) The energy levels and tune-out wavelengths for the $4 s_{1 / 2, F=1}$ state of ${ }^{39} \mathrm{~K}$. The diagram is not to scale. The position of the primary tune-out wavelength is indicated. The tune-out wavelengths for the $4 s_{1 / 2, F=2}$ state are very similar, but there is no tune-out wavelength between the $F=0$ and $F=1$ states of $4 p_{3 / 2}$ while a new tune-out wavelength occurs between the $F=2$ and $F=3$ states.

TABLE VI: Tune-out wavelengths (in $\mathrm{nm}$ ) for the $4 s_{1 / 2, F=1}$ and $4 s_{1 / 2, F=2}$ states of ${ }^{39} \mathrm{~K}$. Calculations are done with $\alpha_{1}$ defined by Eq. (7). The tune-out wavelengths omitting consideration of hyperfine splitting are given for comparison in boldface. Tune-out wavelengths are given to eight digits after the decimal point to ensure differences between adjacent wavelengths near the $4 p_{3 / 2, F^{\prime}}$ states are given to at least two digits. The total uncertainty in the tune-out wavelengths is about 0.001-0.002 $\mathrm{nm}$ as discussed in the text.

\begin{tabular}{cc}
\hline \hline $4 s_{1 / 2, F=1}$ & $4 s_{1 / 2, F=2}$ \\
\hline $\mathbf{7 6 8 . 9 7 0 7 6 0 9 0}$ & $\mathbf{7 6 8 . 9 7 0 7 6 0 9 0}$ \\
770.10782637 & 770.10870313 \\
768.97018480 & 768.97110657 \\
766.70036069 & 766.70125931 \\
766.70034560 & 766.70123125 \\
$\mathbf{4 0 5 . 9 1 7 3 1 4 3 8}$ & $\mathbf{4 0 5 . 9 1 7 3 1 4 3 8}$ \\
405.91716336 & 405.91740499 \\
404.83548479 & 404.83573392 \\
$\mathbf{4 0 4 . 7 2 1 7 6 2 9 4}$ & $\mathbf{4 0 4 . 7 2 1 7 6 2 9 4}$ \\
404.72160507 & 404.72185767 \\
404.52831645 & 404.52856786 \\
404.52831507 & 404.52856533 \\
\hline \hline
\end{tabular}

tween the excitation thresholds of the $4 p_{1 / 2,1}$ and $4 p_{1 / 2,2}$ states. The hyperfine structure associated with the $4 p_{3 / 2}$ state results in two additional tune-out wavelengths located between the three $4 p_{3 / 2, F}$ levels that can undergo a dipole transition with the $4 s_{1 / 2, F^{\prime}}$ hyperfine level.

There is one tune-out wavelength for any transition which we define as the primary tune-out wavelength. 
This wavelength is the one which most closely corresponds to the tune-out wavelengths calculated without hyperfine splitting. For the $F=1$ state of ${ }^{39} \mathrm{~K}$ it is indicated on Figure 1 as the wavelength of $768.970185 \mathrm{~nm}$ which lies between the excitation thresholds of the $4 p_{1 / 2}$ and $4 p_{3 / 2}$ states. The primary tune-out wavelength for the $4 s_{1 / 2, F=2}$ state of ${ }^{39} \mathrm{~K}$ is $768.971107 \mathrm{~nm}$. The absolute precision of these two tune-out wavelength estimates should be about 0.001-0.002 $\mathrm{nm}$ [3]. However, the differences between these two wavelengths is known to a much higher precision. The difference in the energies for these two tune-out wavelengths is very closely related to the hyperfine splitting between these two levels. Table VIII compares the energy differences between the two tune-out primary wavelengths and the hyperfine energy splitting of the two $4 s_{1 / 2}$ hyperfine states. They are in agreement to better than $1.5 \%$ and this implies increased precision in the calculated hyperfine shifts of the tune-out frequencies.

While the calculations of the hyperfine Stark shift are critically reliant on the use of the energy adjusted reduced dipole matrix elements, this is not true for the tune-out wavelengths. For example, the adjustments to the reduced matrix element made a contribution of $1 \times 10^{-6} \mathrm{~nm}$ to the ${ }^{39} \mathrm{~K} \mathrm{~F}=1$ tune-out wavelength of $768.970185 \mathrm{~nm}$. The change in the matrix element contributed $8 \times 10^{-6} \mathrm{~nm}$ to the $405.917163 \mathrm{~nm}$ tune-out wavelength.

This information above suggests a very simple approach to determine the hyperfine splitting of the primary tune-out wavelengths that are located between the two states of the spin-orbit doublet of the first excited state. The frequency difference would just be the energy difference in the $4 s_{1 / 2}$ hyperfine ground states, i.e.

$$
\delta \omega_{\text {tune-out }} \approx \delta E_{\mathrm{hfs}}\left(4 s_{1 / 2}\right)
$$

The difference between the positions of the primary tuneout wavelengths with and without hyperfine splitting is also almost equal to the hyperfine energy shifts. For example, the tune-out frequency omitting hyperfine splitting is 0.0592523862 a.u. The tune-out frequency for the $F=1$ state is 0.0592524306 a.u. The energy difference of $4.44 \times 10^{-8}$ a.u. is almost the same as the hyperfine energy shift of $4.39 \times 10^{-8}$ a.u.

The tune-out wavelengths also depend on the magnetic sub-level of potassium that is occupied. The tune-out wavelengths associated with the different magnetic sublevels of the $4 s_{1 / 2, F}$ states are listed in the supplementary data. The tune-out wavelengths for the $M_{F}=0$ and $M_{F}=1$ states of ${ }^{39} \mathrm{~K}\left(4 s_{1 / 2, F=1}\right)$ were $768.9701808 \mathrm{~nm}$ and $768.9701868 \mathrm{~nm}$ respectively. The differences in the tune-out wavelengths for any of the different magnetic sub-levels do not exceed $2 \times 10^{-5} \mathrm{~nm}$.
TABLE VII: Tune-out wavelengths (in $\mathrm{nm}$ ) for the $4 s_{1 / 2, F=7 / 2}$ and $4 s_{1 / 2, F=9 / 2}$ states of ${ }^{40,41} \mathrm{~K}$. Calculations are done with $\alpha_{1}$ defined by Eq. (77). The tune-out wavelengths omitting consideration of hyperfine splitting are given for comparison in boldface.

\begin{tabular}{cc}
\hline \hline${ }^{40} \mathrm{~K}\left(4 s_{1 / 2, F=7 / 2}\right)$ & ${ }^{40} \mathrm{~K}\left(4 s_{1 / 2, F=9 / 2}\right)$ \\
\cline { 2 - 2 } $\mathbf{7 6 8 . 9 7 0 5 1 2 8 3}$ & $\mathbf{7 6 8 . 9 7 0 5 1 2 8 3}$ \\
770.10942041 & 770.10697923 \\
768.97193896 & 768.96937193 \\
766.70202758 & 766.69955766 \\
766.70195723 & 766.69947243 \\
$\mathbf{4 0 5 . 9 1 7 1 8 5 6 3}$ & $\mathbf{4 0 5 . 9 1 7 1 8 5 6 3}$ \\
405.91755943 & 405.91688660 \\
404.83589207 & 404.83519815 \\
$\mathbf{4 0 4 . 7 2 1 6 3 4 5 5}$ & $\mathbf{4 0 4 . 7 2 1 6 3 4 5 5}$ \\
404.72202530 & 404.72132195 \\
404.52873087 & 404.52803363 \\
404.52872456 & 404.52802596 \\
\hline${ }^{41} \mathrm{~K}\left(4 s_{1 / 2, F=1}\right)$ & $41 \mathrm{~K}\left(4 s_{1 / 2, F=2}\right)$ \\
\hline $\mathbf{7 6 8 . 9 7 0 2 9 5 9 9}$ & $\mathbf{7 6 8 . 9 7 0 2 9 5 9 9}$ \\
770.10759459 & 770.10807699 \\
768.96997907 & 768.97048613 \\
766.70013234 & 766.70062962 \\
766.70012847 & 766.70061632 \\
$\mathbf{4 0 5 . 9 1 7 0 6 5 5 9}$ & $\mathbf{4 0 5 . 9 1 7 0 6 5 5 9}$ \\
405.91698251 & 405.91711543 \\
404.83530536 & 404.83544246 \\
$\mathbf{4 0 4 . 7 2 1 5 1 4 7 9}$ & $\mathbf{4 0 4 . 7 2 1 5 1 4 7 9}$ \\
404.72142795 & 404.72156690 \\
404.52813758 & 404.52827616 \\
404.52813722 & 404.52827496 \\
\hline \hline
\end{tabular}

2. ${ }^{40,41} K$

All properties involving ${ }^{40,41} \mathrm{~K}$ should be interpreted with the contents of the previous section in mind. First of all, the hyperfine Stark shift calculations for these isotopes can expected to have an overall accuracy similar to those of ${ }^{39} \mathrm{~K}$. However, not much significance can attributed to the differences in the polarizabilities of the ${ }^{39,40,41} \mathrm{~K}$ ground states.

The tune-out wavelengths for ${ }^{40} \mathrm{~K}$ and ${ }^{41} \mathrm{~K}$ are given in Table VII. The tune-out wavelengths without any hyperfine splitting are also tabulated. The differences between the tune-out wavelengths for the different hyperfine states is largest for ${ }^{40} \mathrm{~K}$. This is expected since ${ }^{40} \mathrm{~K}$ has the largest hyperfine interaction constants.

The shifts in energies have a much larger effect on the tune-out wavelengths than the changes in reduced matrix elements. So differences between the tune-out wavelengths for different isotopes can be estimated from the data in Tables VI and VII to a precision of about $10^{-5}$ nm. 
TABLE VIII: Comparison of the $4 s_{1 / 2}$ state hyperfine energy splittings and the energy splitting of the primary tune-out frequencies, $\omega_{\text {to }}$ mainly due to hyperfine splitting of the $4 s_{1 / 2}$ level. The notation $a[b]$ means $a \times 10^{b}$.

\begin{tabular}{lcc}
\hline \hline Transition & $\Delta E_{\mathrm{hfs}}$ (a.u.) & $\Delta \omega_{\text {to }}$ (a.u.) \\
\hline${ }^{39} \mathrm{~K} W_{F=2}-W_{F=1}$ & $7.017[-8]$ & $7.103[-8]$ \\
${ }^{40} \mathrm{~K} W_{F=7 / 2}-W_{F=9 / 2}$ & $1.954[-7]$ & $1.978[-7]$ \\
${ }^{41} \mathrm{~K} W_{F=2}-W_{F=1}$ & $3.861[-8]$ & $3.907[-8]$ \\
\hline \hline
\end{tabular}

\section{CONCLUSIONS}

The impact of hyperfine structure on the tune-out wavelengths of three isotopes of potassium have been calculated. The hyperfine structure of the ground and excited states leads to a number of additional tune-out wavelengths. The additional tune-out frequencies associated with the hyperfine splitting of the $n p_{J}$ excited states would be difficult to detect due to the very small energy splittings of the hyperfine levels. The additional tuneout wavelengths associated with the splitting of the $4 \mathrm{~s}$ ground state are more likely to be detectable in an experiment. The splittings in the tune-out wavelengths for the primary tune-out wavelength of ${ }^{39,40,41} \mathrm{~K}$ are $0.0008 \mathrm{~nm}$, $0.0027 \mathrm{~nm}$ and $0.0005 \mathrm{~nm}$ respectively. The uncertainty in a recent experiment was $0.0015 \mathrm{~nm} \mathrm{[1]}$. The hyperfine splitting in the tune-out wavelengths would become ap- parent with a modest increase in experimental precision.

The method used to determine the shifts in the tuneout frequency was unorthodox, being essentially a second order calculation using energy and matrix element shifts applied prior to the evaluation of the oscillator strength sum rules. This simplified calculation was adequate for a system with $(L, J)=(0,1 / 2)$ quantum numbers and a nucleus with a small quadrupole moment and the agreement with a previous all-order MBPT calculation 11. could hardly have been better. The method could be made more rigorous without a great deal of effort. It should be possible to incorporate the hyperfine operator into the Hamiltonian prior to diagonalization. The additional computational efforts would not be prohibitive.

One result of the present analysis is that the tune-out wavelength is largely insensitive to the small changes in the matrix elements resulting from the hyperfine interaction. The hyperfine interaction driven changes in the tune-out wavelengths are most sensitive to the changes in the transition frequencies. Indeed, the dominant effect on the tune-out wavelengths can probably be estimated by just taking into account the energy splits and shifts caused by the hyperfine interaction.

This research was supported by the Australian Research Council Discovery Project DP-1092620. We thank Dr Marianna Safronova for suggesting we investigate the shifts in the tune-out wavelengths caused by the hyperfine interaction.
[1] W. F. Holmgren, R. Trubko, I. Hromada, and A. D. Cronin, Phys. Rev. Lett. 109, 243004 (2012).

[2] B. Arora, M. S. Safronova, and C. W. Clark, Phys. Rev. A 84, 043401 (2011).

[3] J. Jiang, L. Y. Tang, and J. Mitroy, Phys. Rev. A 87, 032518 (2013).

[4] A. D. Cronin (2013), (private communication).

[5] L. J. LeBlanc and J. H. Thywissen, Phys. Rev. A 75, 053612 (2007).

[6] C. D. Herold, V. D. Vaidya, X. Li, S. L. Rolston, J. V. Porto, and M. S. Safronova, Phys. Rev. Lett. 109, 243003 (2012).

[7] N. Bouloufa, A. Crubellier, and O. Dulieu, Phys. Scr. Volume T 134, 014014 (2009).

[8] J. Mitroy, M. S. Safronova, and C. W. Clark, J. Phys. B 43, 202001 (2010).

[9] E. Simon, P. Laurent, and A. Clairon, Phys. Rev. A 57, 436 (1998).

[10] W. R. Johnson, U. I. Safronova, A. Derevianko, and M. S. Safronova, Phys. Rev. A 77, 022510 (2008).

[11] U. I. Safronova and M. S. Safronova, Phys. Rev. A 78, 052504 (2008).

[12] A. Godone, D. Calonico, F. Levi, S. Micalizio, and C. Calosso, Phys. Rev. A 71, 063401 (2005).
[13] K. Beloy, U. I. Safronova, and A. Derevianko, Phys. Rev. Lett. 97, 040801 (2006).

[14] E. J. Angstmann, V. A. Dzuba, and V. V. Flambaum, Phys. Rev. Lett. 97, 040802 (2006).

[15] H. P. Kelly, R. L. Chase, G. R. Daum, and J. J. Chang, Phys. Rev. A 8, 2777 (1973).

[16] T. Lee, T. P. Das, and R. M. Sternheimer, Phys. Rev. A 11, 1784 (1975).

[17] J. L. Snider, Phys. Lett. 21, 172 (1966).

[18] J. R. Mowat, Phys. Rev. A 5, 1059 (1972).

[19] E. Arimondo, M. Inguscio, and P. Violino, Rev. Mod. Phys. 49, 31 (1977).

[20] L. J. Armstrong, Theory of the Hyperfine Structure of Free Atoms (Wiley-Interscience, New York, 1971).

[21] S. Falke, E. Tiemann, C. Lisdat, H. Schnatz, and G. Grosche, Phys. Rev. A 74, 032503 (2006).

[22] Y. Singh, D. K. Nandy, and B. K. Sahoo, Phys. Rev. A 86, 032509 (2012).

[23] J. Ney, Z. Phys. 223, 126 (1969).

[24] A. Behrle, M. Koschorreck, and M. Köhl, Phys. Rev. A 83, 052507 (2011).

[25] M. S. Safronova and W. R. Johnson, Adv. At. Mol. Opt. Phys. 55, 191 (2008). 